\title{
Prolonged Pertussis Outbreak in Ontario Originating in an Under-immunized Religious Community
}

\author{
Deeks $\mathbf{S L}^{1,2^{*}}$, Lim $\mathbf{G H}^{1}$, Walton $\mathbf{R}^{1}$, Fediurek $\mathbf{J}^{1}$, Lam $\mathbf{F}^{1}$, Walker $\mathbf{C}^{3}$, Walters $\mathbf{J}^{4}$ and Crowcroft $\mathbf{N S}^{1,2,5}$
}

1 Public Health Ontario, Toronto, ON

2 Dalla Lana School of Public Health, University of Toronto, Toronto, ON

3 Elgin St. Thomas Public Health

$4 \quad$ Wellington-Dufferin-Guelph Public Health

5 Department of Laboratory Medicine and Pathobiology, Faculty of Medicine, University of Toronto, Toronto,ON

* Corresponding author: shelley.deeks@oahpp.ca

\begin{abstract}
Background: A prolonged pertussis outbreak began in Ontario in November 2011 in an under-immunized religious community and subsequently spread to the general population and a second religious community in the same region of the province.

Objective: To compare the epidemiology in the religious communities to that of the general population within the affected jurisdictions.

Methods: The analysis includes cases reported through the integrated Public Health Information System (iPHIS) between November 1, 2011 and April 15, 2013 that met the outbreak case definition. Health unit staff assessed whether cases were members of religious communities through case investigations and collected information on immunization status, treatment and outcomes.
\end{abstract}

Results: A total of 443 confirmed and probable outbreak cases were reported in 7 health units. The outbreak began in one religious community (138 cases), before spreading to the general population in the region (273 cases). A second under-immunized community within the region experienced 32 cases. Thirteen cases were hospitalized and no deaths were reported. Disease peaked earlier in the religious community; cases were significantly younger, more likely to be at high risk for pertussis and more likely to be unimmunized. Among the fully immunized general population, $51 \%$ of cases were between 10-14 years and with a median of 5.6 years since their last immunization.

Conclusion: The epidemiology of pertussis in the under-immunized community is distinct from the general population. Transmission of pertussis to the general community is not unexpected during an outbreak; however, the proportion of cases up to date with immunization warrants further investigation.

\section{Introduction}

Between 2010 and 2012, a number of jurisdictions throughout North America were experiencing pertussis outbreaks. ${ }^{1-5}$ Pertussis is a highly communicable respiratory infection caused by Bordetella pertussis, which is most dangerous in infants under one year of age; particularly those under four months. ${ }^{6,7}$ Disease control is challenging due to the long period of communicability, the disease's atypical presentation among adults and immunized children and the fact that it can be difficult to diagnose. ${ }^{8,9,10}$

Pertussis vaccines have been available in Ontario since 1943. In 1984, a combination adsorbed whole cell pertussis vaccine was used in the province's universal publicly-funded immunization program. In 1997, acellular pertussis vaccine replaced the whole cell vaccine and was delivered as part of a combination product (diphtheria [D], tetanus [T], acellular pertussis [aP], inactivated polio vaccine [IPV] and Haemophilus influenzae type b [Hib]) at 2, 4, 6 months of age, with a pentavalent (DTaP-IPV-Hib) booster dose at 18 months and a quadrivalent 
booster dose (DTaP-IPV) at 4-6 years of age. In 2003, an adolescent dose was publicly-funded using dTap at 1416 years of age. Finally, in 2011, a single dose adult pertussis immunization program was introduced. ${ }^{11}$

On January 25, 2012 a provincial outbreak of pertussis was declared after a cluster of cases was detected in an under-immunized religious community (religious community A) in southwestern Ontario, Canada. The outbreak was initially limited to three health units (HUs) and one religious community (A) but subsequently spread to the general community and a second under-immunized religious community $(B)$ in 7 HUs in the province.

Our objective is to report on the outbreak investigation, comparing the epidemiology in the religious communities to that of the general population, including demographics, immunization status, treatment and outcomes.

\section{Methods}

\section{Surveillance}

Pertussis is a legally reportable disease in Ontario (population 13.5 million in 2012). ${ }^{12}$ Cases are identified passively and case information is entered into a provincial reportable diseases registry, the integrated Public Health Information System (iPHIS). Pertussis outbreak case definitions which included a geographic component were established and modified three times throughout the course of the outbreak to reflect the evolving situation. The outbreak period was between November 1, 2011 and April 15, 2013. We extracted data entered into iPHIS meeting the confirmed and probable pertussis outbreak case definitions on August 22, 2013. Extracted data included demographic information, laboratory results, hospitalization status, outcome, and immunization history, although completeness varied by field. We determined episode date for each case using a standardized hierarchy: symptom onset date was preferentially selected, specimen collection date if symptom onset was unavailable, and case reported date if both were unavailable.

Early in the outbreak, we supplemented iPHIS data with additional case note data using an enhanced case report form to gather information on whether the case was at high risk for pertussis, as well as health seeking behavior and treatment. A manual case record review was undertaken on cases reported between November 1, 2011 and August 15, 2012. We classified persons as high risk for pertussis if they were pregnant or under 1 year of age, and assessed whether cases obtained laboratory testing, initiated antibiotic therapy and the time interval to seek treatment.

HUs were requested to identify connection(s) to under-immunized communities within iPHIS. We classified cases as being from one of two under-immunized religious communities, $A$ and $B$, using specific exposure identifiers. For some analyses, religious communities $A$ and $B$ were combined. We assumed cases not assigned to either exposure identifier originated from the remaining $\mathrm{HU}$ population, (the general population).

As accurately assessing immunization status from a single field in iPHIS was problematic, we reviewed the following fields for all cases: immunization agent, immunization administration dates, relevant immunizations upto-date (RI), and risk factor (RF). The number of doses of pertussis-containing vaccine received was established based on the number of administration dates reported for each case. We determined the validity of doses by considering minimum age and minimum interval requirements between doses, as per the Ontario publicly funded schedule. ${ }^{13}$ An interval of at least 14 days prior to disease onset was required for a dose to be considered valid. We assessed cases without any valid doses (i.e., no administration dates) as unimmunized if $\mathrm{Rl}=\mathrm{No}$ or $\mathrm{RF}=\mathrm{unimmunized;}$ otherwise we assessed these cases as having unknown immunization status. We assessed cases with at least one valid dose as being complete for age (i.e., up to date) or partially immunized in accordance with the provincial immunization schedule and guidelines established by the National Advisory Committee on Immunization. ${ }^{11,14}$

\section{Statistical analysis}

We calculated incidence rates per 100,000 population using demographic data from Statistics Canada obtained through IntelliHealth Ontario. Population data by HU were unavailable at the time of analysis for 2013; 2012 population data were substituted. Descriptive analyses and statistical tests were performed using SPSS version 
19 (IBM, Armonk, NY, USA) and SAS version 9.2. We compared median age and time intervals for selected outcomes between communities using the Kruskal-Wallis test; statistical significance was declared at $p<0.05$. Where appropriate, we excluded missing data from analyses.

\section{Results}

\section{Descriptive epidemiology}

A total of 443 pertussis cases were reported by 7 outbreak-affected HUs during the outbreak period. Of these, 344 cases $(77.7 \%)$ were classified as confirmed based on laboratory confirmation of pertussis or an epidemiologic link to a laboratory-confirmed case and the remaining 99 were classified as probable based on symptomatology. Cases ranged in age from 14 days to 68 years, with a median age of 9.4 years (age was missing for 6 cases). Females accounted for $56.0 \%$ of cases. The annualized incidence rate during the outbreak was 25.7 cases per 100,000 population (2012 population of $7 \mathrm{HUs}$ was 1.2 million people). The overall provincial rate during the same time period was 6.1 pertussis cases per 100,000 population. Thirteen cases (2.9\%) were hospitalized and there were no deaths. All hospitalized cases were children and 8 were under one year of age.

Of the 443 cases, $31.2 \%$ originated in religious community $A, 7.2 \%$ in religious community $B$ and $61.6 \%$ in the general population (Table 1). The age distribution of cases from the two religious communities was distinct from that of the general population (Figure 1). The median age of cases from the general population was approximately 10 years older and significantly higher than the median age of cases from either of the two religious communities $(p<0.001)$. While over half of the cases from religious community A and B were under 5 years of age, only $19.8 \%$ of the general population cases occurred in this age group $(p<0.0001)$. Among the general population, the highest proportion of cases $(28.2 \%)$ occurred in the 10-14 year age group. The probability of hospitalization did not vary by community.

Table 1: Comparison of selected outcomes among cases from religious community A, B and the general population using iPHIS and case report form data

\begin{tabular}{|l|l|l|l|}
\hline \multicolumn{2}{|c|}{ Religious community A } & \multicolumn{1}{|c|}{ General population } & Religious community B \\
\hline iPHIS Data ( $\mathbf{n}=\mathbf{4 4 3})$ & $138(31.2 \%)$ & $273(61.6 \%)$ & $32(7.2 \%)$ \\
\hline Number of cases (\%) & 3.7 & 13.2 & 3.9 \\
\hline Median age (years) & $60.6 \%$ & $19.8 \%$ & $59.4 \%$ \\
\hline Proportion less than 5 years old & & & 0 \\
\hline Number of forms completed & 115 & $5.3 \%$ & N/A \\
\hline$\%$ high risk & $22.6 \%$ & $81.3 \%$ & N/A \\
\hline$\%$ obtaining laboratory tests & $50.9 \%$ & 188 & 0 \\
\hline
\end{tabular}

$\mathrm{N} / \mathrm{A}=$ not applicable 
Figure 1: Pertussis Cases by Age and Community Status: Ontario, November 1, 2011 to April 15, $2013\left(n=437^{*}\right)$

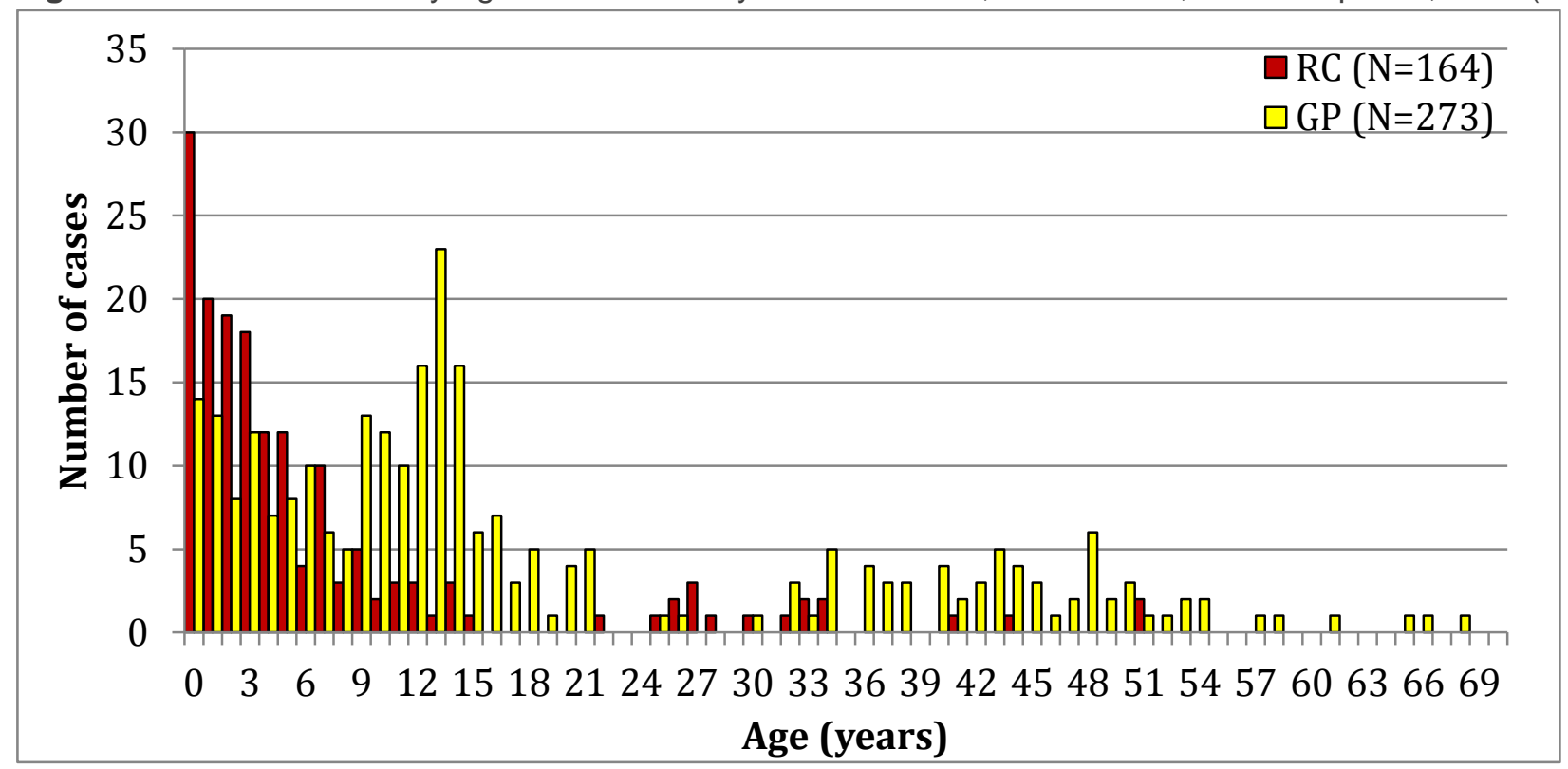

$\mathrm{RC}=$ religious communities $\mathrm{A}$ and $\mathrm{B} ; \mathrm{GP}=$ general population

${ }^{*}$ excludes 6 cases for whom age was unknown.

Enhanced case report forms were available for $68.4 \%$ of cases; 115 from religious community $A$ and 188 from the general population (no cases from religious community $B$ had enhanced case report forms as they occurred later in the outbreak). As seen in Table 1, religious community $A$ cases were more likely than general population cases to be high risk $(p<0.0001)$, and were less likely to obtain the recommended laboratory testing for pertussis $(p<0.0001)$, as well as initiate antibiotic therapy $(p<0.01)$. Religious community $A$ cases also had a longer time period between symptom onset and initiation of treatment compared to the general population cases $(p<0.005)$.

The temporal distribution of cases showed a bimodal pattern over the course of the outbreak (Figure 2). The first wave peaked in January 2012 and was limited almost exclusively to cases from religious community A. The second wave peaked in June 2012 and was comprised primarily of cases from the general population, although the tail of the epidemic curve was mixed and involved religious community $\mathrm{B}$ cases. 
Figure 2: Confirmed and probable outbreak cases $(n=443)$ by onset date, case classification and community, southwestern Ontario: November 1, 2011 to April 15, 2013

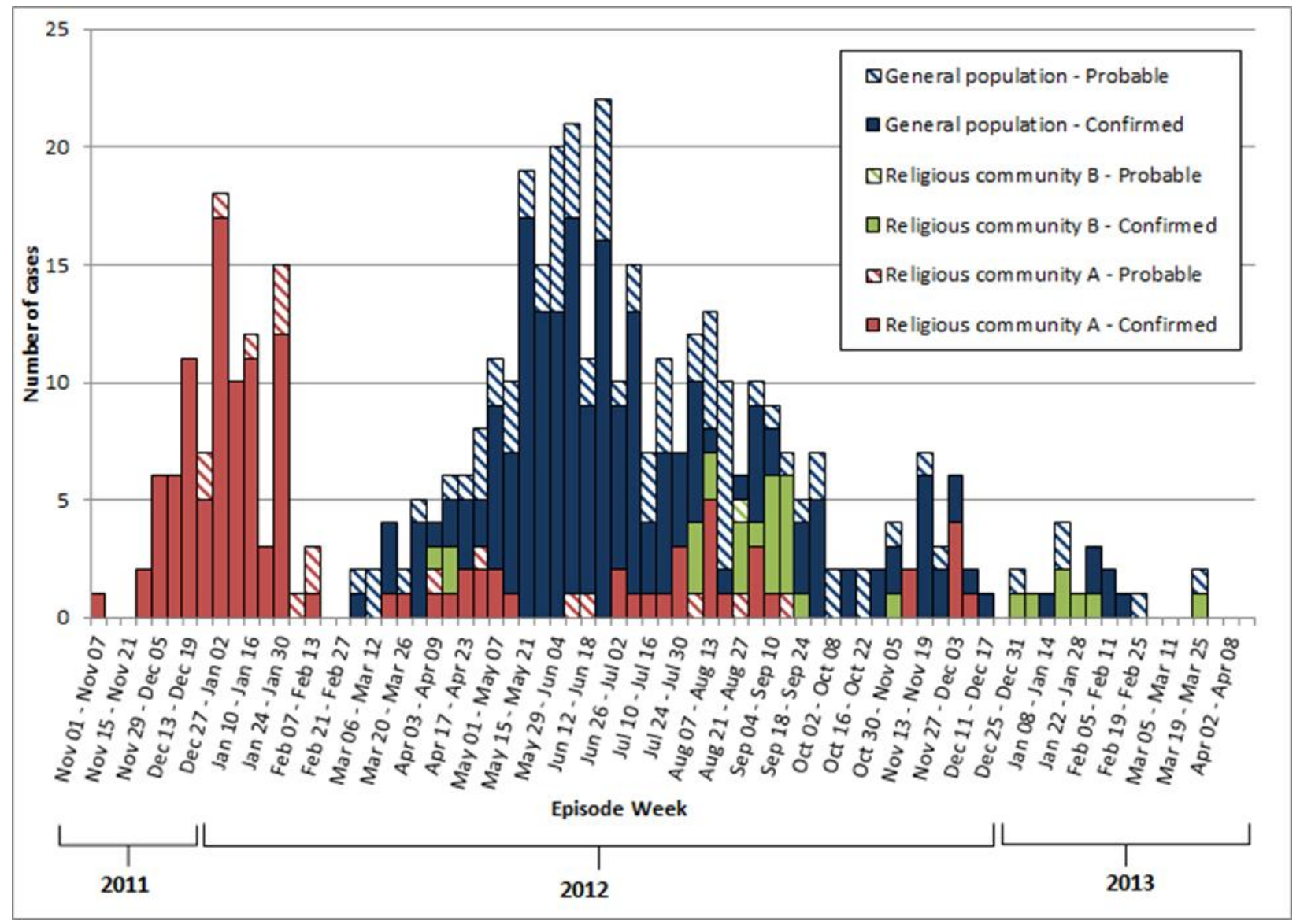

\section{Immunization status}

We could determine immunization status for $85.1 \%$ ( $n=377$ ) of cases. Of these, $34.5 \%$ were complete-for age, $11.9 \%$ were partially immunized, and $53.6 \%$ were unimmunized. Immunization status was similar between the two religious communities and we therefore combined their data for this analysis. We found a significant difference in proportion immunized and complete-for-age between the religious communities and the general population $(p<$ 0.01). While $86.6 \%$ of cases in the two religious communities with known status were unimmunized, only $32.0 \%$ of cases in the general population were unimmunized. The age distribution of unimmunized cases varied by community. Unimmunized cases were younger in the religious communities compared to the general population (median age of 3.0 versus 8.4 years, respectively, $p<.0001)$. Conversely, $6.0 \%(n=9)$ of cases with known immunization status were complete for age in the religious communities compared to $53.1 \%(n=121)$ among the general population $(p<.0001)$. The median age of cases in the general population who were complete for age was 11.7 years (range 2 months to 43 years) and over half (51.2\%) were between $10-14$ years. The median number of years since their last immunization was 5.6 (range 15 days to 9.9 years).

\section{Discussion}

Ontario experienced an outbreak of pertussis that lasted more than 17 months. We found that the epidemiology of pertussis in the under-immunized community was distinct from that of the general population, with a younger age distribution, a larger proportion of cases with no immunization and a larger proportion of high risk cases. The pertussis epidemiology in the under-immunized community is more consistent with that found in the developing world and similar to that seen prior to immunization programs in North America. ${ }^{15,16}$ Despite the younger age 
distribution, the disease did not appear to be more severe in the under-immunized communities. Although $86.6 \%$ of the religious community cases were unimmunized, some community members were willing to receive immunizations during specific outreach clinics held by the HUs, where testing and chemoprophylaxis were also offered.

One concern raised by public health staff arose from the observation that some religious community members declined follow-up for confirmatory laboratory testing. While cases from the religious community were less likely to have laboratory testing than cases from the general population, it was somewhat reassuring that almost half sought testing. It was also encouraging that the majority of individuals from all communities obtained treatment once they were identified as cases, although religious community cases obtained this less often. The delay in receipt of treatment by the religious community may be amenable to public health interventions.

Transmission to the general community is not unexpected during a pertussis outbreak, especially considering the infectiousness of the organism. Ontario does not have a comprehensive immunization registry, so we cannot determine immunization coverage in the general population or vaccine effectiveness. The lack of denominator information for the religious communities also precludes assessment of vaccine effectiveness using the screening method. However, Ontario has a school-based immunization information system and in 2011/2012 school year, the proportion of 7 and 17 year old Ontario students who were up to date with their pertussis immunizations was $76.0 \%$ and $67.7 \%$, respectively. ${ }^{17}$ This coverage is not high enough to prevent transmission of pertussis within communities, especially in regions where coverage is even lower as a result of religious or conscientious objection to immunization.

The proportion of general population cases who were fully immunized in this outbreak was concerning and has also been seen in other recent outbreaks in North America. ${ }^{1,4}$ In Ontario, acellular pertussis vaccine was implemented in 1997, and therefore the oldest cohort of children vaccinated solely with acellular vaccine would have been between approximately 14 and 15 years of age during this outbreak. Although children 10-14 years of age account for $5.7 \%$ of Ontario's population, they accounted for $20.4 \%$ of outbreak cases overall, $28.2 \%$ of the general population cases and $51.2 \%$ of the general population cases who were up to date with their immunizations. This warrants further investigation and is suggestive of waning immunity associated with acelluar pertussis vaccine, which has also been demonstrated in the United States. ${ }^{1}$

Our analysis has a number of limitations. Accurately assessing immunization status within iPHIS is challenging for diseases such as pertussis with a complex immunization schedule. Records were frequently missing clinical information and immunization status was unknown for $14.9 \%$ of cases, despite extensive follow-up by health unit staff. These limitations were somewhat offset through the use of enhanced case report forms at the beginning of the outbreak. Given the prolonged nature of the outbreak and the human resources required to complete the report forms, they were only completed on cases occurring during the first nine and a half months of the outbreak, which may impact their representativeness. Finally, as noted above, we were not able to calculate vaccine effectiveness as we do not have a comprehensive provincial immunization registry.

\section{Conclusion}

This outbreak provided the opportunity to contrast pertussis epidemiology in distinct communities within one geographic area. Transmission from under-immunized communities to the general community is not unexpected, however the number of adolescent cases who were up to date with immunization suggests waning vaccine immunity. 


\section{References}

1. Klein NP, Bartlett J, Fireman B, et al. Comparative Effectiveness of Acellular Versus Whole-Cell Pertussis Vaccines in Teenagers. Pediatrics. 2013 Jun;131(6):e1716-22. doi: 10.1542/peds.2012-3836. Epub 2013 May 20.

2. Pharmaceutical Services Division, Ministry of Health British Columbia. Pertussis Outbreak Management in Fraser Health and Vancouver Coastal Health Authorities. http://www.health.gov.bc.ca/pharmacare/pdf/5d.pdf

3. Center for Disease Control and Prevention. Pertussis Outbreak Trends. http://www.cdc.gov/pertussis/outbreaks/trends.html

4. Office of the Chief Medical Officer of Health, New Brunswick, Canada. Whooping Cough in New Brunswick. http://www2.gnb.ca/content/gnb/en/departments/ocmoh/cdc/content/whooping_cough.html .

5. Center for Disease Control and Prevention. Pertussis Outbreak Trends. Available at: http://www.cdc.gov/pertussis/outbreaks/trends.html.

6. von König CH, Halperin S, Riffelmann M, Guiso N. Pertussis of adults and infants. Lancet Infect Dis. 2002 Dec;2(12):744-50.

7. Guiso N. Bordetella pertussis: Why is it still circulating? J Infect. 2013 Oct 5. doi:pii: S0163-4453(13)00289-2. 10.1016/j.jinf.2013.09.022. [Epub ahead of print].

8. Deeks S, De Serres G, Boulianne N, Duval B, Rochette L, Dery P, et al. Failure of physicians to consider the diagnosis of pertussis in children. Clin Infect Dis. 1999;28(4):840-6.

9. Taylor ZW, Ackerson B, Bronstein DE, Lewis K, Steinberg E, Stone MM, Viraraghavan R, Wong VK, Salzman MB. Wheezing in Children with Pertussis Associated with Delayed Pertussis Diagnosis. Pediatr Infect Dis J. 2013 Oct 28. [Epub ahead of print].

10. Cornia PB, Hersh AL, Lipsky BA, Newman TB, Gonzales R. Does this coughing adolescent or adult patient have pertussis? JAMA. 2010 Aug 25;304(8):890-6. doi: 10.1001/jama.2010.1181.

11. Ontario Ministry of Health \& Long-Term Care. Publicly funded immunization schedules for Ontario.

August 2011.

http://www.health.gov.on.ca/en/public/programs/immunization/docs/schedule.pdf

12. Ministry of Health \& Long-Term Care: Appendix B: Provincial Case Definitions for Reportable Disease: Pertussis. Ontario Public Health Standards: Infectious Diseases Protocol, 2009. http://www.health.gov.on.ca/en/pro/programs/publichealth/oph_standards/infdispro.aspx

13. Ontario Ministry of Health \& Long-Term Care. Recommended and minimum ages and intervals between vaccine doses of publicly funded routine childhood vaccines. August 2011 version 2. http://www.health.gov.on.ca/en/pro/programs/immunization/docs/age_interval.pdf

14. National Advisory Committee on Immunization (NACl). Pertussis vaccine. Canadian Immunization Guide. Evergreen ed. Ottawa: Public Health Agency of Canada; 2012. http://www.phac-aspc.gc.ca/publicat/cig-gci/p04-pert-coqu-eng.php

15. Gordon JE, Hood RI. Whooping cough and its epidemiological anomalies. Am J Med Sci. 1951 Sep;222(3):333-61.

16. Crowcroft NS, Pebody RG. Recent developments in pertussis. Lancet. 2006 Jun 10;367(9526):1926-36.

17. Wilson S, Lim GH, Fediurek J, McIntyre MA, Deeks SL. 2011/12 Immunization coverage from Ontario, Canada: uptake and exemptions. National Foundation for Infectious Diseases' 16th Annual Conference on Vaccine Research, Baltimore, USA, April 22-24, 2013 [oral presentation].

18. Tartof SY, Lewis M, Kenyon C, et al. Waning immunity to pertussis following 5 doses of DTaP. Pediatrics. 2013 Apr;131(4):e1047-52. doi: 10.1542/peds.2012-1928. Epub 2013 Mar 11.

19. Klein NP, Bartlett J, Rowhani-Rahbar A, Fireman B, Baxter R. Waning protection after fifth dose of acellular pertussis vaccine in children. N Engl J Med. 2012 Sep 13;367(11):1012-9. doi: 10.1056/NEJMoa1200850. 


\section{Acknowledgements}

The authors would like to thank all persons involved in the outbreak and outbreak response from the affected Health Units, the Ontario Ministry of Health and Long Term Care and Public Health Ontario.

\section{Conflict of Interest}

All authors have no conflicts of interest to disclose.

\section{Funding}

No external funding was secured for this study.

\section{Financial Disclosure}

All authors have no financial relationships relevant to this article to disclose. 